\title{
Highlights from High Energy Neutrino Experiments at CERN
}

\author{
W.-D. Schlatter \\ CERN, CH-1211 Geneva, Switzerland \\ dieter.schlatter@cern.ch
}

\begin{abstract}
Experiments with high energy neutrino beams at CERN provided early quantitative tests of the Standard Model. This article describes results from studies of the nucleon quark structure and of the weak current, together with the precise measurement of the weak mixing angle. These results have established a new quality for tests of the electroweak model. In addition, the measurements of the nucleon structure functions in deep inelastic neutrino scattering allowed first quantitative tests of QCD.
\end{abstract}

\section{Introduction}

High energy neutrino beams were used successfully in the 1970s and 1980s to study the weak interaction as well as probing the nucleon with deep inelastic scattering experiments without the interference of strong interaction. At CERN's PS accelerator this was highlighted with the discovery of neutral currents with the Gargamelle heavy liquid bubble chamber experiment ${ }^{1}$ in 1973. In the late 1970s, with even higher energy neutrino beams up to $200 \mathrm{GeV}$, new opportunities opened up for experiments using deep inelastic neutrino-nucleon scattering to test the foundations of the Standard Model which had been formulated in the decade before. This article recalls the highlights from the CERN neutrino experiments at that time. ${ }^{a}$ Historic reviews of early neutrino experiments by D. H. Perkins and J. Steinberger can be found in Refs. 3 and 4 respectively.

Deep inelastic neutrino-nucleon scattering is usually described by four kinematic variables, $Q^{2}, \nu, x$ and $y$; for convenience their definitions are repeated in Fig. 1.

The neutrino and antineutrino cross-sections are described by three nucleon structure functions, $2 x F_{1}\left(x, Q^{2}\right), F_{2}\left(x, Q^{2}\right)$, and $x F_{3}\left(x, Q^{2}\right)$. In the Parton Model spin $1 / 2$ partons imply $2 x F_{1}(x)=F_{2}(x)$ and with $q(x)$ and $\bar{q}(x)$ the sum of all quark and antiquark structure functions, the cross-sections depend on only two structure functions: $F_{2}(x)=q(x)+\bar{q}(x)$ and $x F_{3}(x)=q(x)-\bar{q}(x)$.

\footnotetext{
${ }^{a}$ The story of the discovery of neutral currents by Gargamelle is recalled in a separate article in this book. ${ }^{2}$
} 


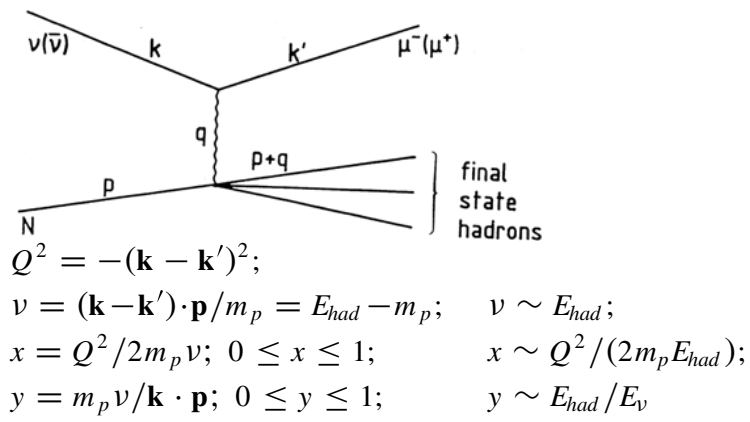

Fig. 1. Definition of kinematic variables in deep inelastic neutrino-nucleon scattering.

\section{Early Gargamelle Results on the Quark Parton Model}

In the late 1960 s the Parton Model $^{5}$ was formulated by R. Feynman, prompted by the new SLAC electron-nucleon scattering experiments. ${ }^{\mathrm{b}}$ The observed scaling behaviour was best explained by point-like constituents of the nucleon, called partons. One of the important elements of the Parton Model is the idea that "scaling" of deep inelastic lepton-nucleon scattering is understood as the sum of elastic scatterings of the lepton on free partons within the nucleon. As a consequence the structure functions of the nucleon scale, i.e., $F_{i}\left(x, Q^{2}\right) \rightarrow F_{i}(x)$ in the limit of very large $Q^{2}$ and $\nu$ with $x$ fixed.

In the early 1970s deep inelastic neutrino-nucleon scattering experiments made with Gargamelle, the heavy liquid bubble chamber at the CERN PS, could clarify some of the open questions. Two important observations were made. Firstly, a linearly rising cross-section with energy in deep inelastic neutrino and antineutrino interactions $^{7}$ confirming the evidence for point-like constituents of the nucleon (see Fig. 2). ${ }^{\mathrm{c}}$

Secondly, the structure function $F_{2}$ from the Gargamelle neutrino data ${ }^{8}$ agrees with $F_{2}$ from the ep scattering experiment at SLAC, ${ }^{9}$ when divided by a charge factor $5 / 18, F_{2}^{\nu N}=F_{2}^{e N}\left[\frac{1}{2}\left((2 / 3)^{2}+(1 / 3)^{2}\right)\right]^{-1}$, the mean square charge of the $\mathrm{u}$ and $\mathrm{d}$ quarks in the nucleon, as predicted by the Quark Model for fractionally charged quarks. The point-like partons are really quarks. This is illustrated in Fig. 3 showing $F_{2}$ from Gargamelle neutrino data as a function of the scaling variable $x$ compared to parametrisations of the SLAC/MIT electron-proton data.

Furthermore, two important sum rules of the Quark Parton Model were also evaluated by the Gargamelle experiment. The momentum sum rule,

\footnotetext{
${ }^{\mathrm{b}}$ A personal recollection by J. I. Friedman of those experiments can be found in Ref. 6 .

${ }^{\mathrm{c}}$ Actually, a linearly rising cross-section in neutrino scattering had been observed before in a heavy liquid bubble chamber experiment at $\mathrm{CERN}^{10}$ "but the significance had not been appreciated at the time". 11
} 


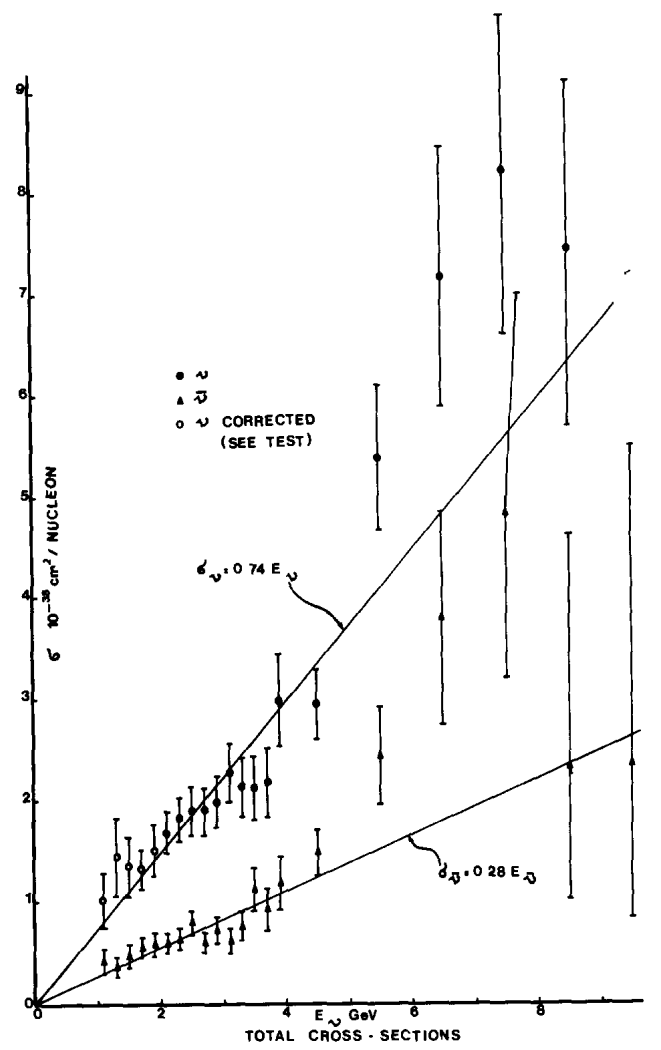

Fig. 2. Total cross-section for neutrino and antineutrino scattering as a function of energy. ${ }^{7}$ The linear rise is a consequence of the point-like interaction of the constituents.

$\frac{1}{2} \int\left(F_{2}^{\nu p}(x)+F_{2}^{\nu n}(x)\right) d x=0.49 \pm 0.07$, in good agreement with the earlier results from the electron scattering, implied that the momentum fraction carried by quarks in the nucleon is about $1 / 2$ of the nucleon momentum, indicating the existence of a new partonic constituent, the gluon. In addition, the number of valence quarks in the nucleon $=\frac{1}{2} \int\left(F_{3}^{\nu p}(x)+F_{3}^{\nu n}(x)\right) d x$ was measured to be $3.2 \pm 0.6$, consistent with the Quark Model expectation of three.

\section{Neutrino Beams and Experiments}

Progress in deep inelastic neutrino scattering experiments came with higher energy neutrino beams and larger, more powerful detectors. At CERN, high energy neutrino beams became available with the construction of the SPS, which was completed by 1976 and the first neutrino beam was commissioned in December of that year.

There were two types of neutrino beams at the SPS, a narrow band beam (NBB), using momentum selected charged hadrons (pions and kaons) and a 


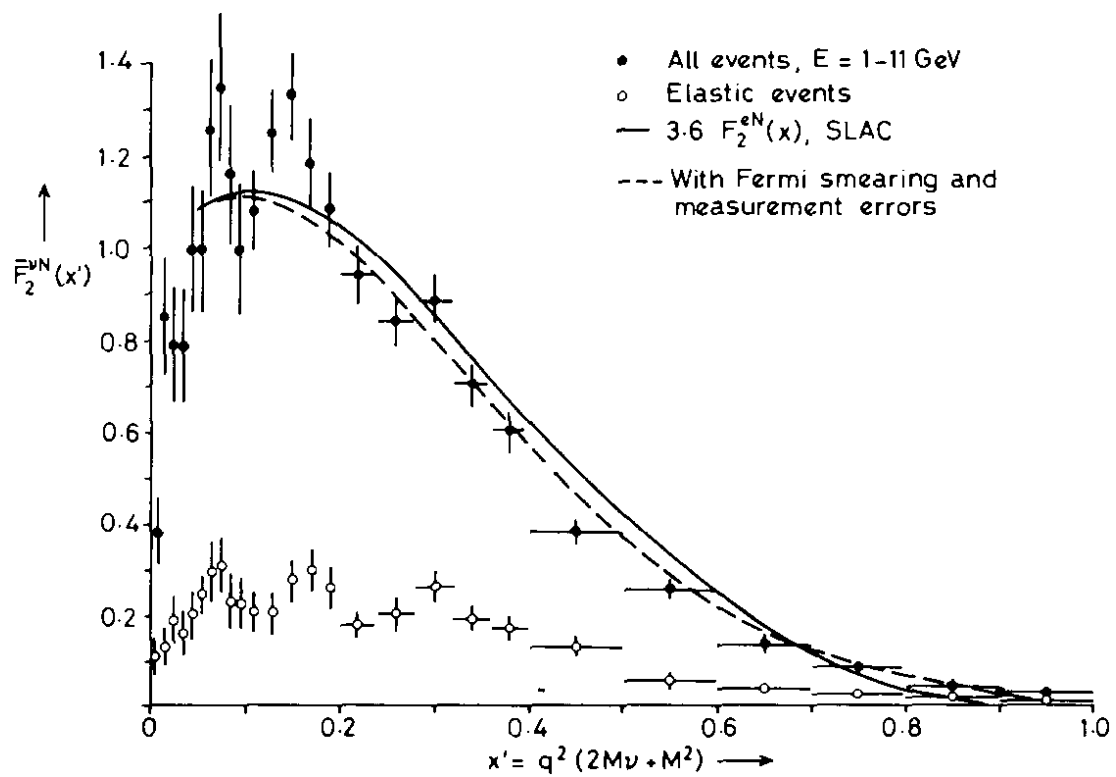

Fig. 3. The structure function $F_{2}(x)$ for neutrino scattering from Gargamelle. ${ }^{7}$ The curves show empirical fits of quark momentum distributions from electron scattering, ${ }^{9}$ multiplied by 18/5.

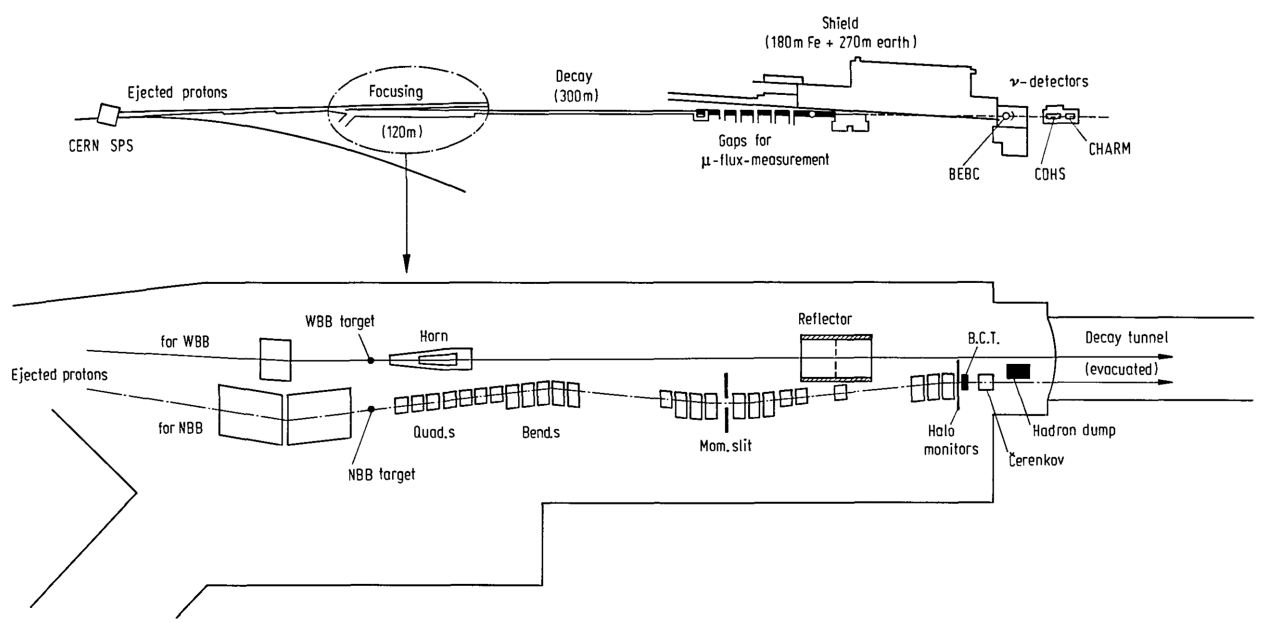

Fig. 4. Layout of the SPS neutrino beams. The lower half shows the focussing part enlarged. ${ }^{12}$

more intense wide-band beam (WBB), using van der Meer focussing horns. A layout of the neutrino area is shown in Fig. 4 and the spectra of these beams for neutrinos and antineutrinos are shown in Fig. 5. Positive hadrons produce neutrinos, negative ones antineutrinos. Narrow band beams permit the determination of the energy of the events, also of neutral currents, using the radial 


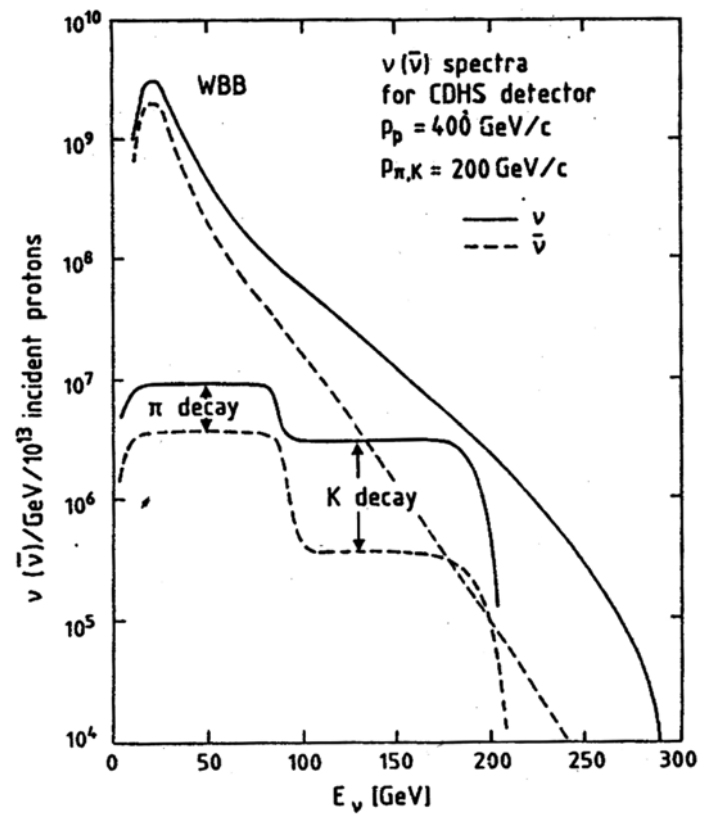

Fig. 5. Fig. 5. Neutrino and antineutrino energy spectra of the wide band beam (falling spectra) and the narrow band beam (flat spectra).

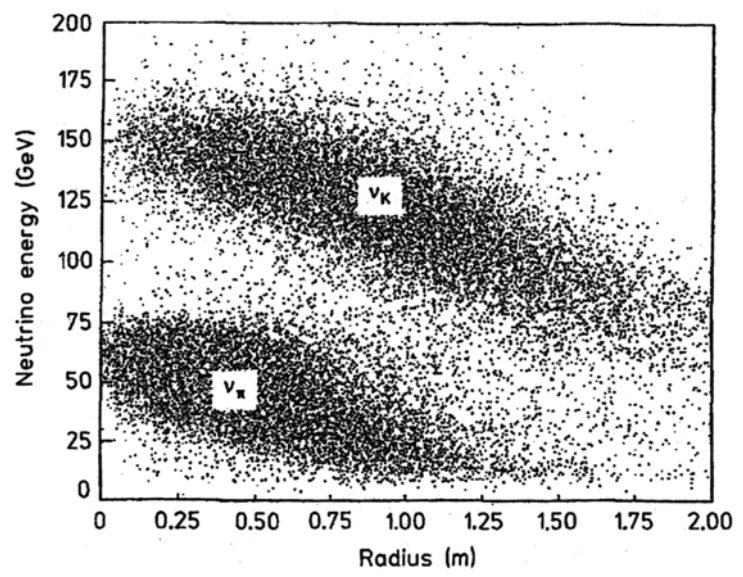

Fig. 6. Narrow band beam energy vs. radius of events in the detector, top group from kaon decay, bottom from pion decay.

position of the event in the detector. This is shown for charged current events in Fig. 6.

After the $300 \mathrm{~m}$ long decay tunnel and the $400 \mathrm{~m}$ long iron shield, followed four detectors: the Big European Bubble Chamber, BEBC, which could be filled with hydrogen, deuterium or neon, and the two new electronic detectors, CDHS, 
and CHARM. The fourth detector was Gargamelle which was moved from the PS neutrino beam to the SPS beams in 1977. However, only one year later the experiment had to be terminated due to a crack in the chamber body.

The CDHS detector ${ }^{13}$ combined the function of target, hadron calorimeter, and muon spectrometer integrally in 19 similar modules, forming a scintillator calorimeter with toroidally magnetised iron plates as absorber. Between the modules were drift chambers for track reconstruction. The total weight was $1200 \mathrm{t}$; the detector started data taking in Spring 1977. The layout is shown in Fig. 7. The second electronic detector, CHARM, ${ }^{14}$ consisted of a fine grained calorimeter surrounded by a magnetised iron frame followed by a muon spectrometer. The calorimeter was composed of scintillators, drift and streamer tubes in between marble absorber plates. The total weight was $100 \mathrm{t}$ and the detector layout is shown in Fig. 8.

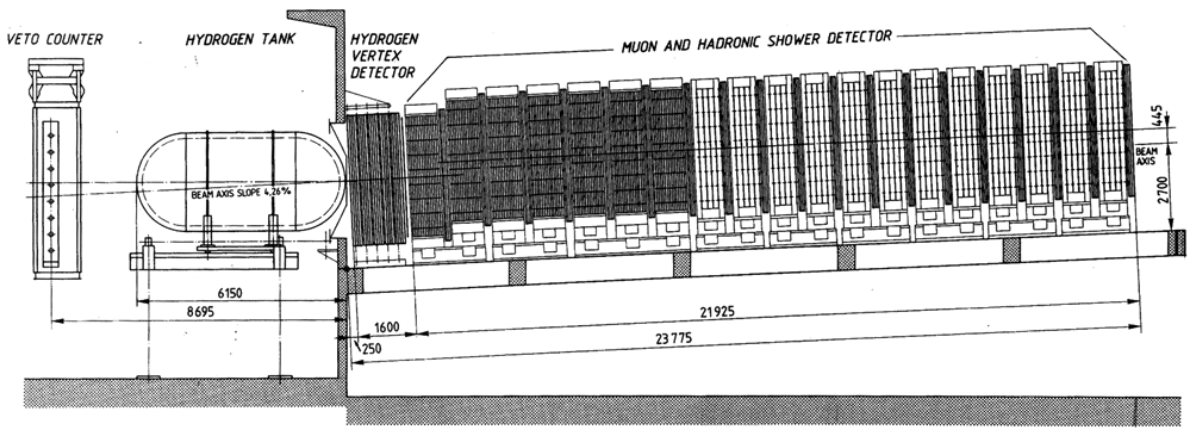

Fig. 7. Layout of the CDHS detector. ${ }^{13}$

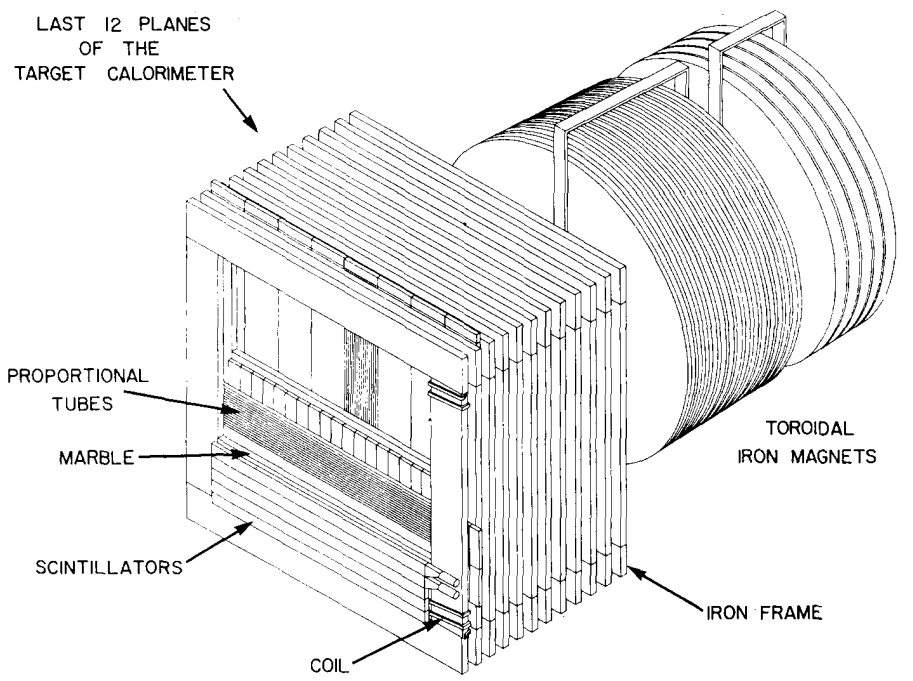

Fig. 8. Layout of the CHARM detector. ${ }^{14}$ 

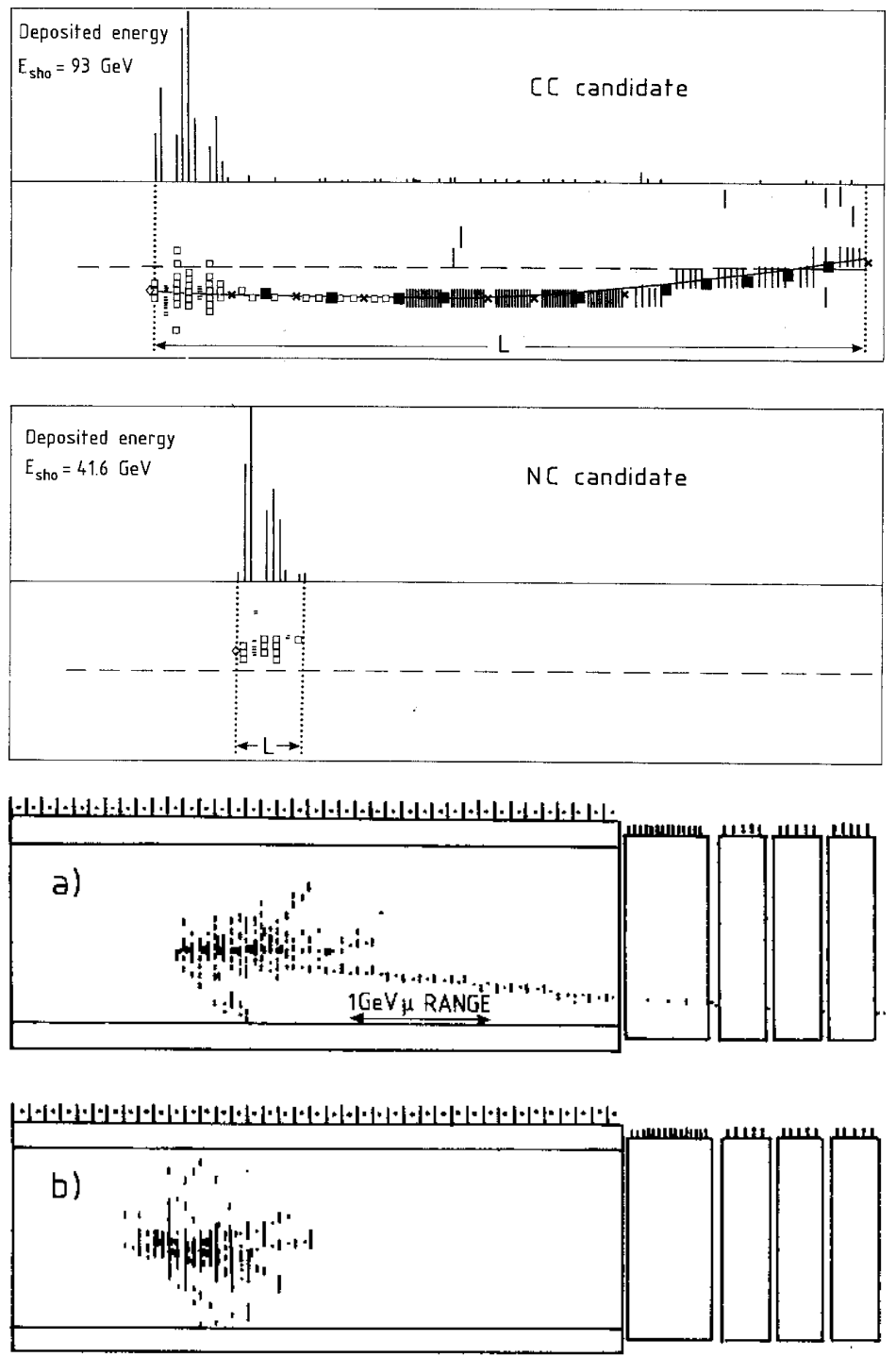

Fig. 9. Displays of CC and NC events in the CDHS (top) and CHARM (bottom) detectors.

Typical charged-current (CC) and neutral-current (NC) events in these detectors are shown in Fig. 9.

\section{Nuclear Structure and Quark Parton Model}

In the late 1970s, high energy neutrino data were collected at the CERN SPS with narrow band beams for which neutrino fluxes could be measured much 


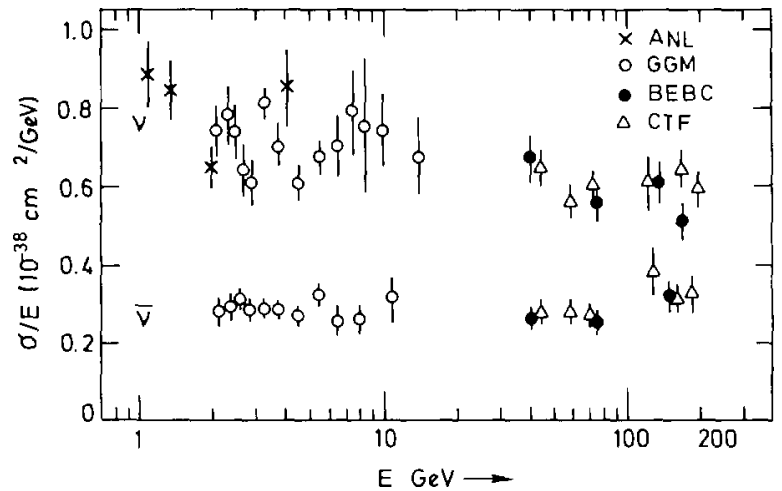

Fig. 10. Results on total cross-sections, $(\sigma / E)$, for neutrinos and antineutrinos from $\mathrm{BEBC}^{17}$ and Gargamelle. ${ }^{15,} 16$ Data from the Caltech-Fermilab and ANL 12-foot BC experiments are also shown.

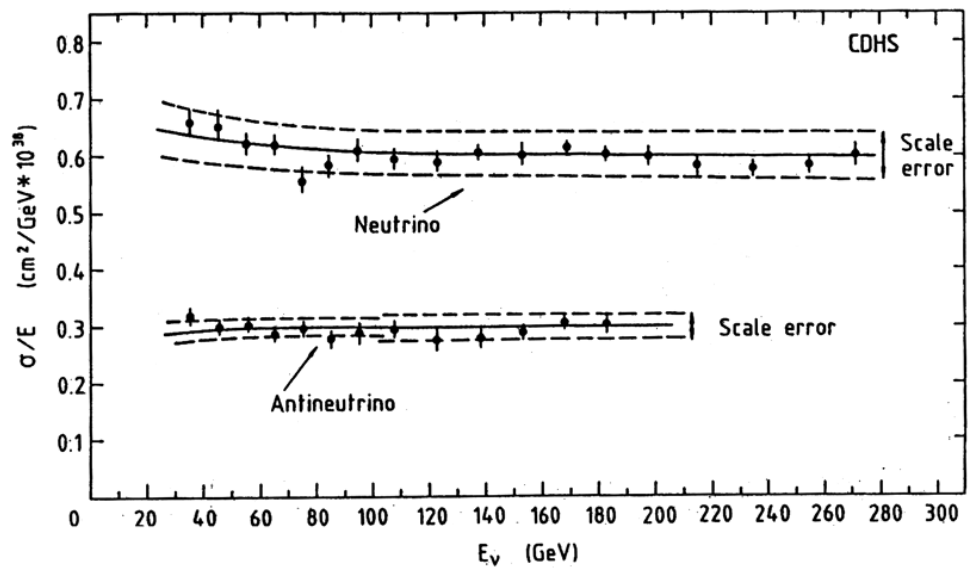

Fig. 11. Total cross-sections, divided by the neutrino energy, for neutrinos and antineutrinos, ${ }^{18}$ illustrating scaling behaviour in the Parton Model.

more reliably. Cross-section measurements at the SPS have been presented from $\mathrm{BEBC}^{17}$ and $\mathrm{CDHS},{ }^{18}$ the most precise data came from the $1200 \mathrm{t}$ calorimeter of CDHS. Results are shown in Fig. 10 for BEBC and Fig. 11 for CDHS. The high energy behaviour of the ratio $\sigma / E$ illustrates the Parton Model prediction of scaling. The expected scaling violation from QCD is too small in this energy range $(<5 \%)$ to be seen. The $y$ distribution, $y$ being approximately equal to the relative hadron energy, is another convenient way to compare to the predictions of the Parton Model. Figure 12 illustrates a remarkable agreement with the Quark Parton Model assumption of point-like structure of the nucleon. 


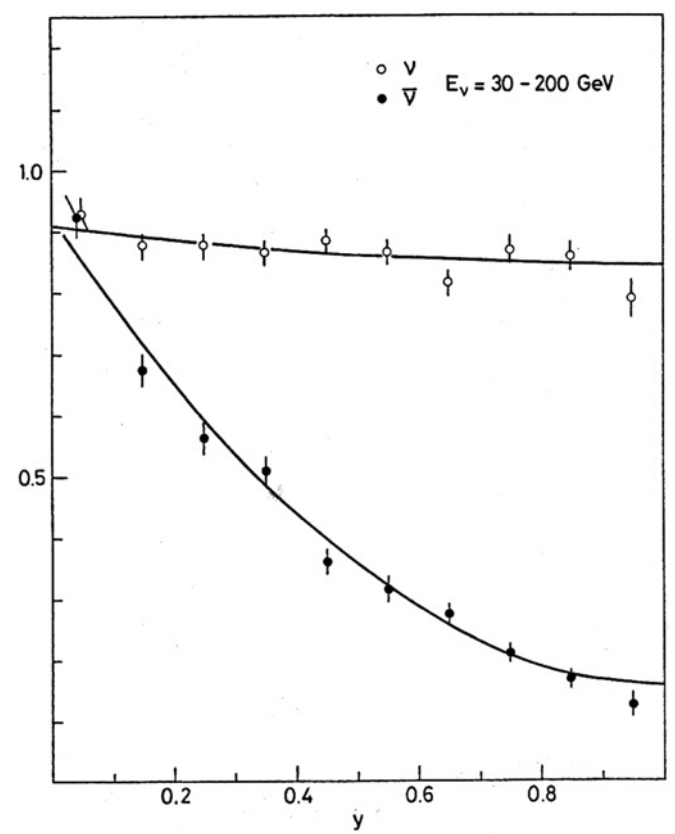

Fig. 12. CDHS results for the $y$ distributions for neutrino and antineutrino deep inelastic scattering. ${ }^{21}$ The lines are the predictions of the Quark Parton Model.

The structure function $F_{2}(x)$ measured with high statistics data has been compared for neutrinos from $\mathrm{CDHS}^{18}$ with results from $e N$ scattering from SLAC$\mathrm{MIT}^{20}$ with a charge factor of $18 / 5$ and with $\mu N$ scattering from EMC $^{19}$ with a charge factor of $9 / 5$. Figure 13 shows that the notion of quarks being the point-like partons could be further strengthened.

\section{Electroweak Measurements}

\subsection{Weak mixing angle}

After the discovery of the neutral current interaction by the Gargamelle experiment ${ }^{1}$ interest was concentrated on measurements of its strength and structure.

From the ratios of neutral to charged current inclusive cross-sections for neutrinos $\left(R_{\nu}\right)$ and antineutrinos $\left(R_{\bar{\nu}}\right)$ the electroweak mixing angle, called the "Weinberg angle", can be extracted within the electroweak theory. During the years 1974 to 1976 Gargamelle presented first estimates of the Weinberg angle, the result was $\sin ^{2} \theta_{W}=0.3-0.4$. During $1977 \mathrm{CDHS}$ has measured $R_{\nu}$ and $R_{\bar{\nu}}$ with high statistics data collected with the NBB at the SPS. CDHS was able to extract a first precise measurement of the Weinberg angle with the value of $\sin ^{2} \theta_{W}=0.24 \pm 0.02$ (Fig. 14). 


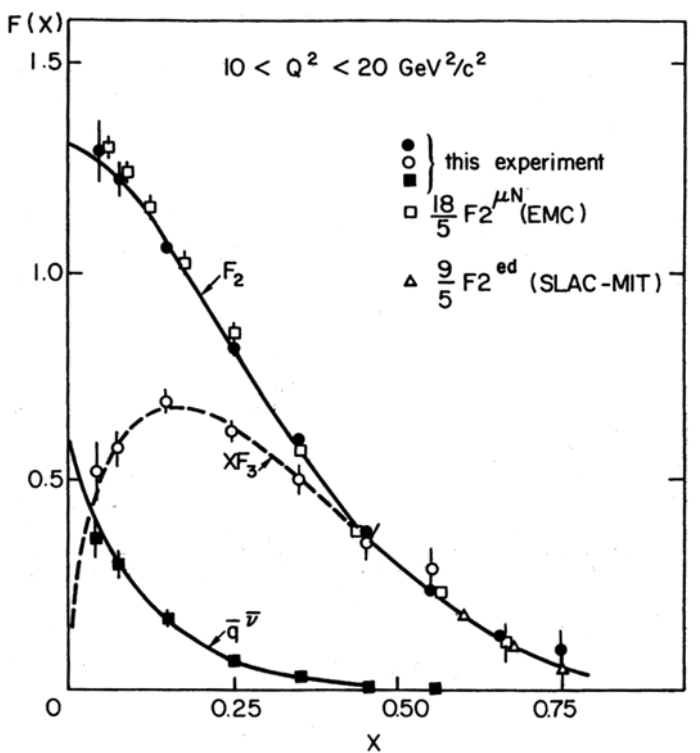

Fig. 13. CDHS results for the structure functions $F_{2}(x), x F_{3}(x)$ and $\bar{q}(x)$, the sum of all antiquark structure functions. ${ }^{18}$ Superimposed for $F_{2}$ are the results for $\mu N$ and ed scattering, multiplied with the corresponding charge factors. The lines are the predictions of the Quark Parton Model.

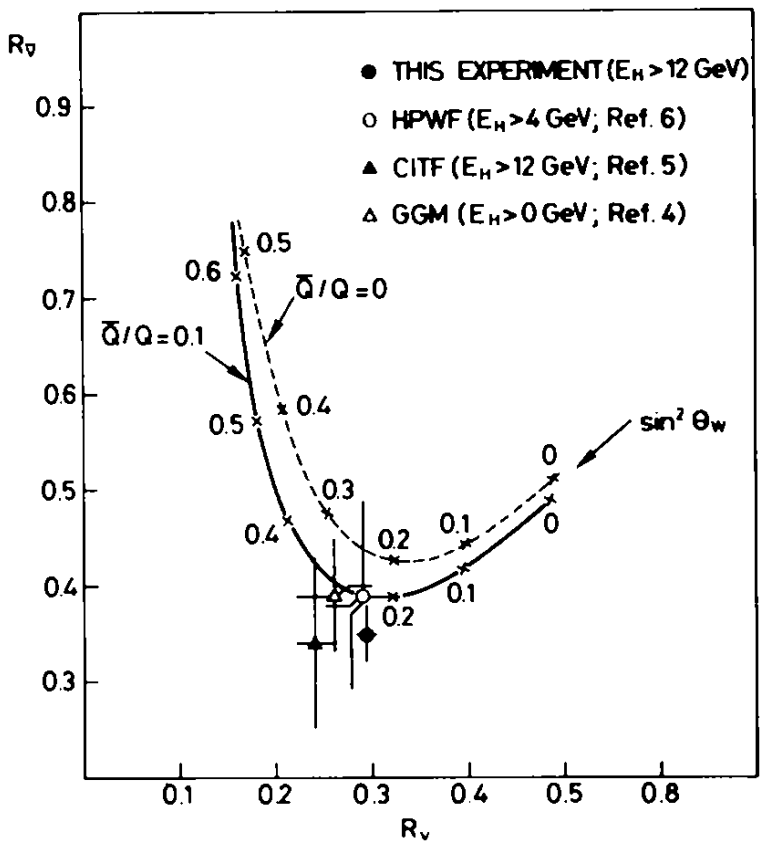

Fig. 14. CDHS cross-section ratios ${ }^{22} R_{\nu}$ and $R_{\bar{\nu}}$ compared to the Weinberg-Salam model. An antiquark contribution of $\bar{q} / q=0.1$ (solid line) was assumed and, for comparison, $\bar{q} / q=0$ (dashed line). 


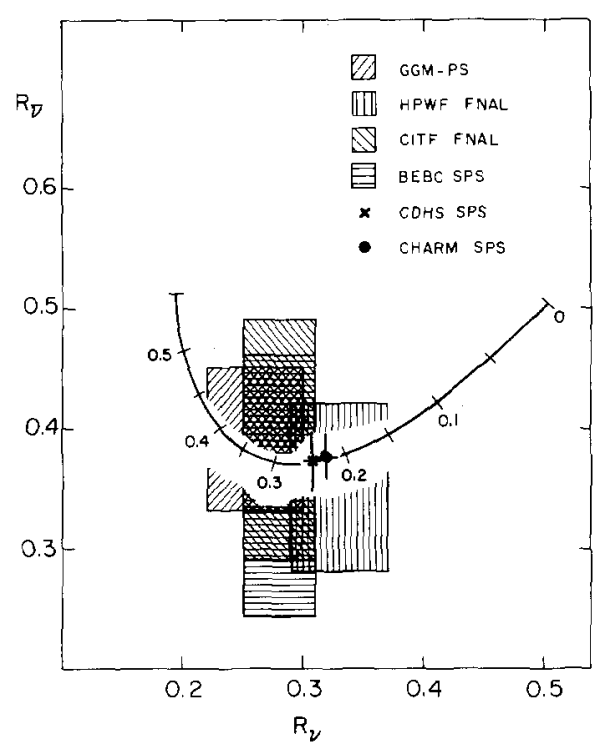

Fig. 15. CHARM cross-section $\operatorname{ratios}^{23} R_{\nu}$ and $R_{\bar{\nu}}$ compared to the Weinberg-Salam model.

Table 1 Various measurements of the weak mixing angle from the CDHS and CHARM experiments.

\begin{tabular}{lc}
\hline & $\sin ^{2} \theta_{W}$ \\
\hline CDHS $1977^{22}$ & $0.24 \pm 0.02$ \\
CHARM $1981^{23}$ & $0.230 \pm 0.023$ \\
CDHS $1986^{24}$ & $0.225 \pm 0.006+0.013\left(m_{c}-1.5 \mathrm{GeV}\right)$ \\
CHARM $1986^{25}$ & $0.236 \pm 0.006+0.012\left(m_{c}-1.5 \mathrm{GeV}\right)$ \\
\hline
\end{tabular}

A few years later, the CDHS measurement was nicely confirmed by the CHARM experiment (Fig. 15). The results from both experiments are listed in Table 1. The comparison with the earlier Gargamelle results is visualised in Fig. 16, it illustrates the progress made by the large electronic detectors since the earlier bubble chamber results.

The precision of the analysis was improved with more statistic and the introduction of QED radiative corrections ${ }^{27}$ in the analysis. The dominant uncertainty in the measurement of the Weinberg angle in neutrino scattering became the poor knowledge of the value of the charm quark mass, $m_{c}$. Therefore, the results were presented as function of $m_{c}$ (see Table 1).

\subsection{Charm production and GIM mechanism}

Oppositely charged dimuon events provide access to open charm production, as predicted by the GIM mechanism ${ }^{28}$ for production and semi-leptonic decay 


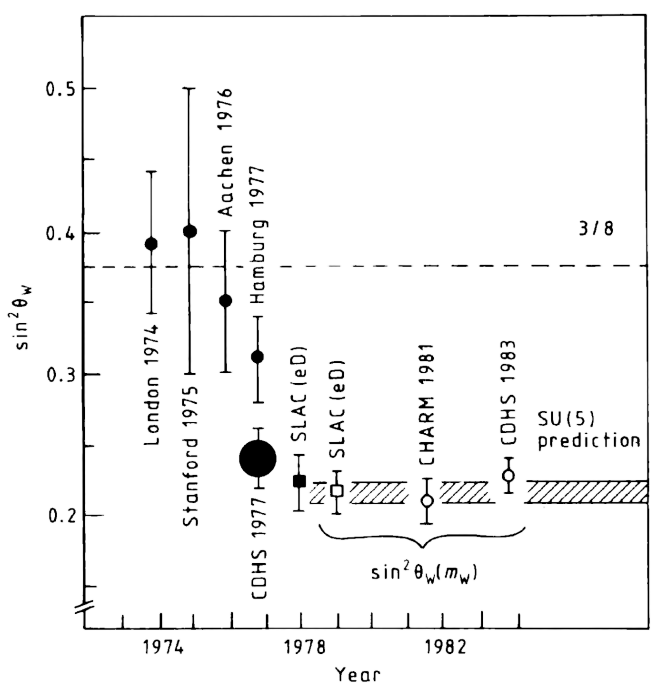

Fig. 16. Different results of early measurements of the Weinberg mixing angle as function of time. ${ }^{26}$ Before 1977, the Gargamelle results are shown.

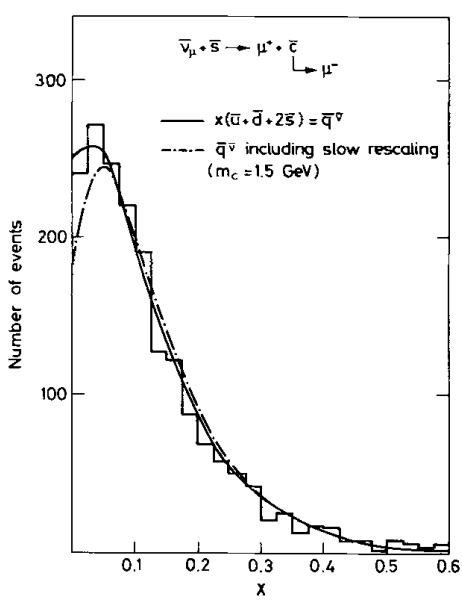

(a)

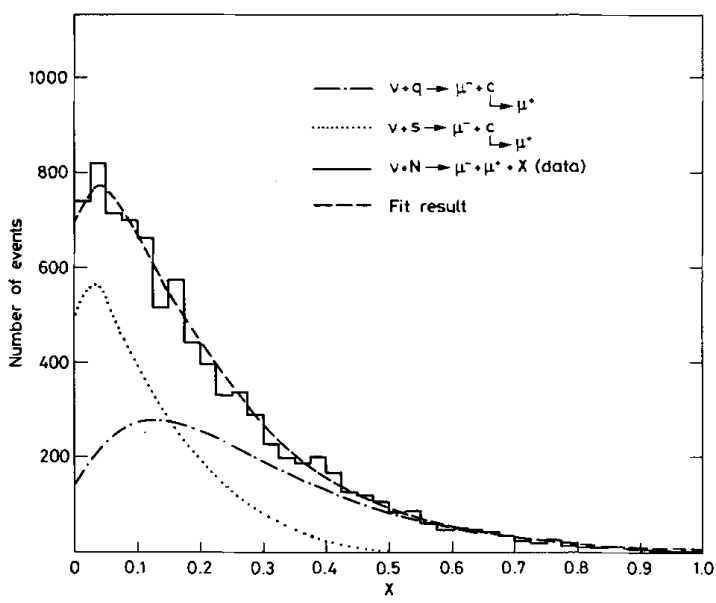

(b)

Fig. 17. Distribution of the $x$ variable for dimuon events. ${ }^{29}$ (a) Antineutrino, the solid curve is the "sea" distribution, $\bar{q}(x)$, from single muon events. (b) Neutrino, the curves show the decomposition into $48 \%$ strange-sea from the data of (a) (dotted curve) and $52 \%$ quark contribution (dashed dotted curve). The dashed curve is the sum.

of charmed particles. The $x$-distribution of dimuons are different from ordinary $\mathrm{CC}$ events and agree well with the specific mixture of quark and antiquark distributions (see Fig. 17). As expected for heavy quarks ${ }^{30}$ charm fragmentation turned out to be hard with an average relative momentum $\langle z\rangle \approx 0.7$. 


\section{QCD and Structure Functions}

The theory of strong interactions of quarks and gluons, QCD, and the notion of "asymptotic freedom" was formulated in 1972/73. Neutrino deep inelastic scattering on the nucleon provided an excellent opportunity for quantitative tests. The analysis of the nucleon structure functions was used to test QCD in detail, to determine the scale parameter $\lambda_{\mathrm{QCD}}$ and the gluon momentum distribution in the nucleon, $g(x)$.

During 1977/78 BEBC and the CDHS experiments showed that the scaling of the naive Quark Parton Model is violated for higher $Q^{2}$. The effect from radiation of hard gluons from the quarks in QCD leads to logarithmic scaling violations and the shape of the nucleon structure function $F_{2}$ is dependent on the neutrino energy (see Fig. 18) with a rise at small $x$ for higher energies and a drop for low energies. Similarly, the $Q^{2}$ dependence at small and large $x$ could clearly be seen in the Gargamelle/BEBC data ${ }^{17}$ (Fig. 19) and the early CDHS data ${ }^{31}$ (Fig. 20).

One of the few cases in which QCD makes an absolute prediction which could be experimentally tested early on is for the structure function $F_{3}$. The moments of the $x$-distribution of $x F_{3}$ (defined as $M_{n}\left(Q^{2}\right)=\int d x x^{n-2} x F_{3}\left(x, Q^{2}\right)$ ) have a simpler $Q^{2}$ dependence in QCD than the distributions themselves, they are predicted to vary as $\log Q^{2}$ to a certain power, called anomalous dimension. In a 2-dimensional $\log -\log$ representation different pairs of moments plotted for different values of $Q^{2}$ should

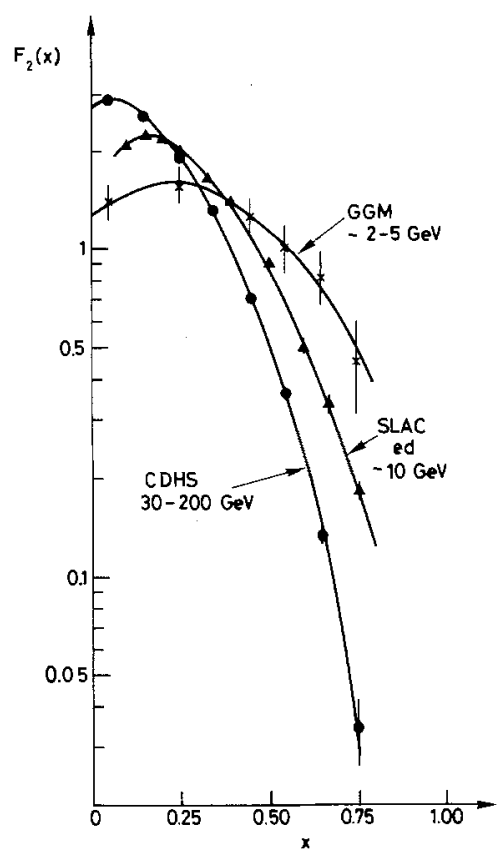

Fig. 18. Comparison of $F_{2}$ structure function seen in different lepton energy domains. ${ }^{31}$ 


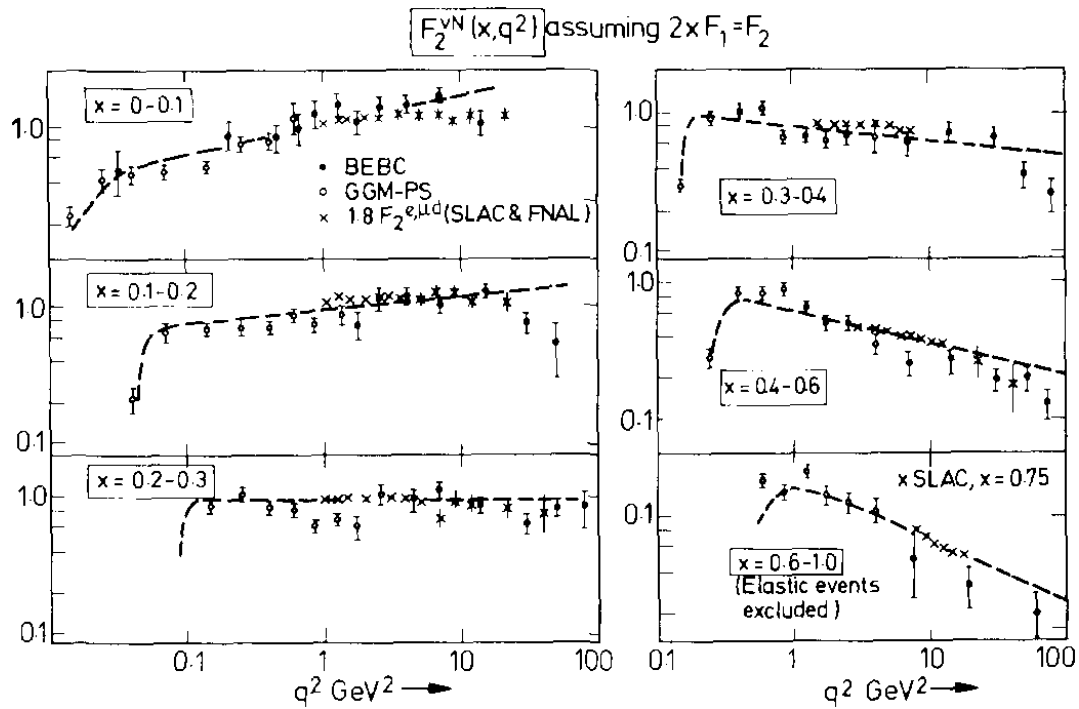

Fig. 19. Gargamelle and BEBC results $F_{2}^{\nu}$ for various $x$ ranges versus $Q^{2} \cdot{ }^{17}$ Results from the SLAC electron and muon scattering experiments, multiplied by the quark charge factor $9 / 5$ are shown by crosses.

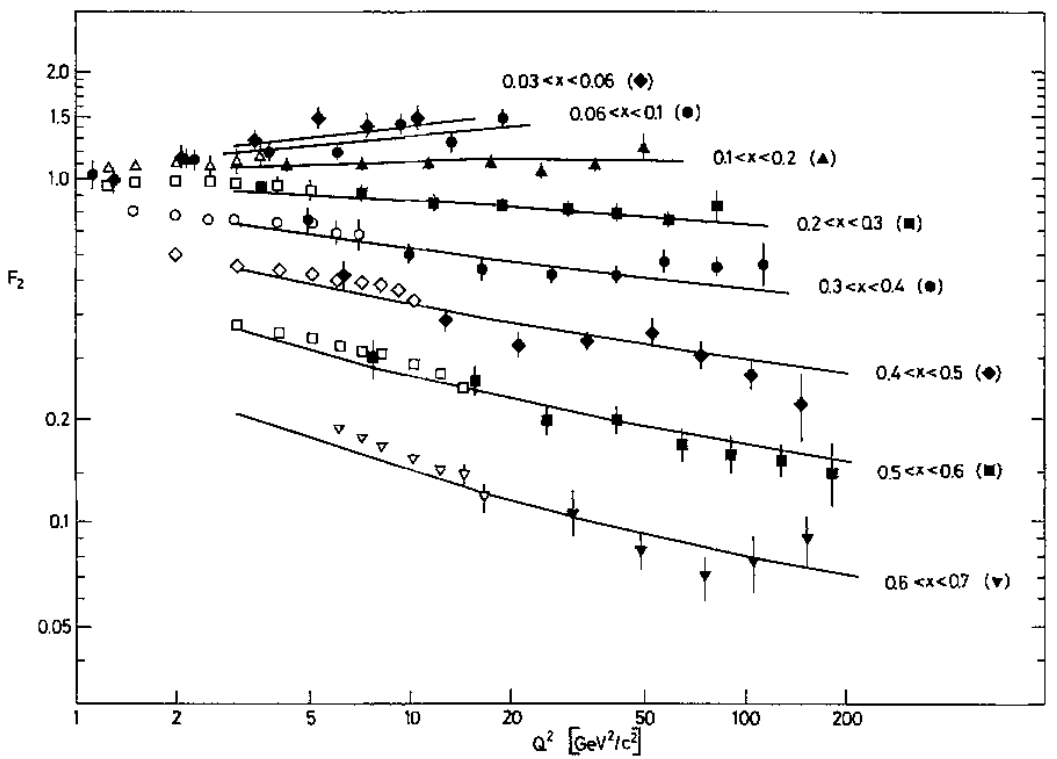

Fig. 20. First CDHS results on $F_{2}\left(x, Q^{2}\right)^{31}$ (solid symbols), with the fits of the DGLAP evolution equations superimposed. Open symbols are for ed scattering. 


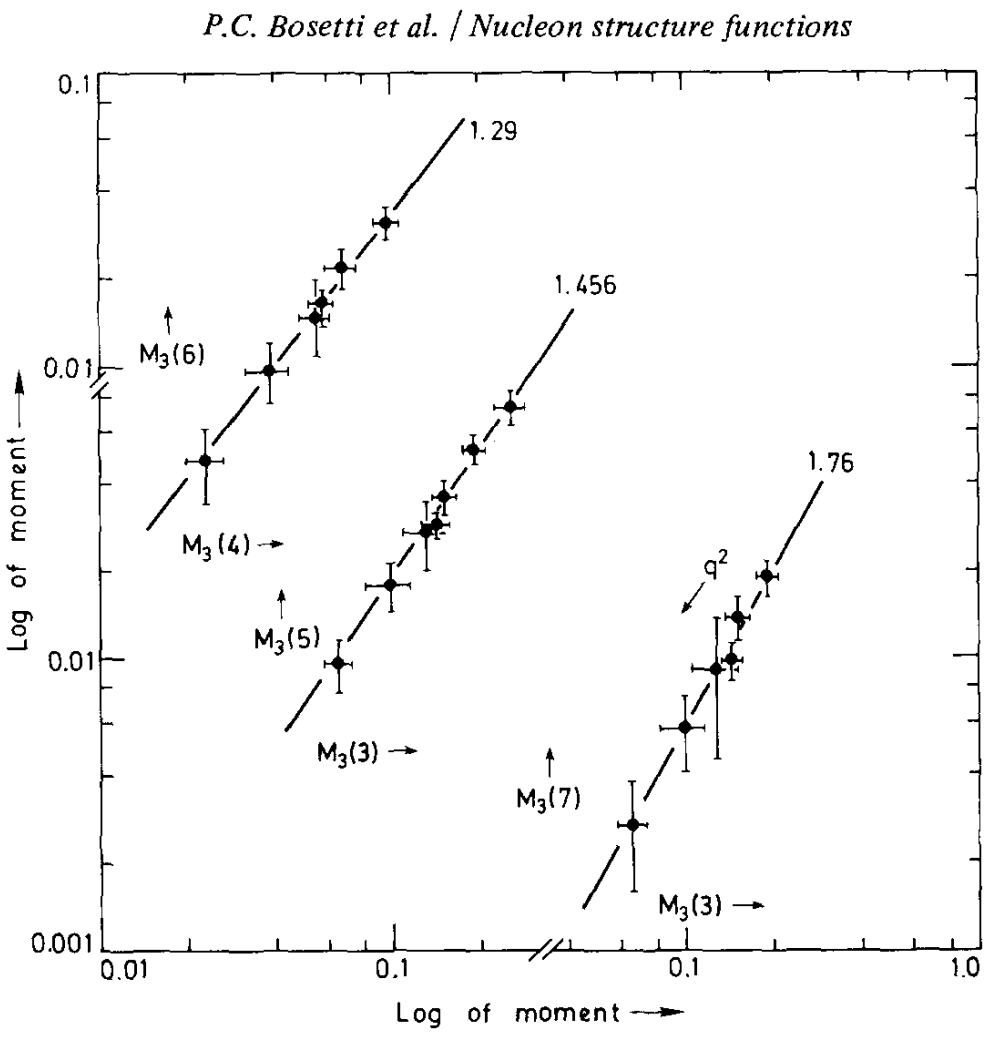

Fig. 21. Log- $\log$ plots of various moments of $x F_{3} \cdot{ }^{17} \mathrm{QCD}$ predicts a linear relation with the slopes indicated. The logarithm of one moment is plotted against another as $Q^{2}$ varies over the range $5-50 \mathrm{GeV}^{2} / c^{2}$. Note, the errors on any pair of moments are strongly correlated.

lie on a straight line with a slope given by the ratio of the anomalous dimensions. This is shown in Fig. 21. The agreement of the observed slopes with the QCD expectation is remarkable, in spite of the relatively low $Q^{2}$.

More stringent quantitative tests of perturbative QCD became possible with more precise high energy neutrino and antineutrino data of $F_{2}$ and $x F_{3}$. In Fig. 22 $F_{2}\left(x, Q^{2}\right)$ and $x F_{3}\left(x, Q^{2}\right)$ are shown for the CDHS data. Fits of the DGLAP evolution equations ${ }^{33}$ for a scale parameter $\lambda_{\mathrm{QCD}}=250 \mathrm{MeV}$ describe well the observed $Q^{2}$ evolution. The logarithm of the scale parameter is related to the running coupling constant of QCD. ${ }^{\mathrm{d}}$ For the typical $Q^{2}$ range of the data from $3 \mathrm{GeV}^{2} / c^{2}$ to $200 \mathrm{GeV}^{2} / c^{2}$ and $\lambda_{\mathrm{QCD}}=250 \mathrm{MeV}$ the corresponding strong coupling constant drops from 0.30 to 0.20 .

Gluons do not take part directly in the deep inelastic neutrino-nucleon scattering process. QCD predicts that their interactions with quarks inside the nucleon leads

$\overline{\mathrm{d}} \alpha_{s}\left(Q^{2}\right)=\frac{12 \pi}{33-2 N_{f}} / \ln \left(Q^{2} / \lambda_{\mathrm{QCD}}^{2}\right), N_{f}$ is the number of quark flavors. 

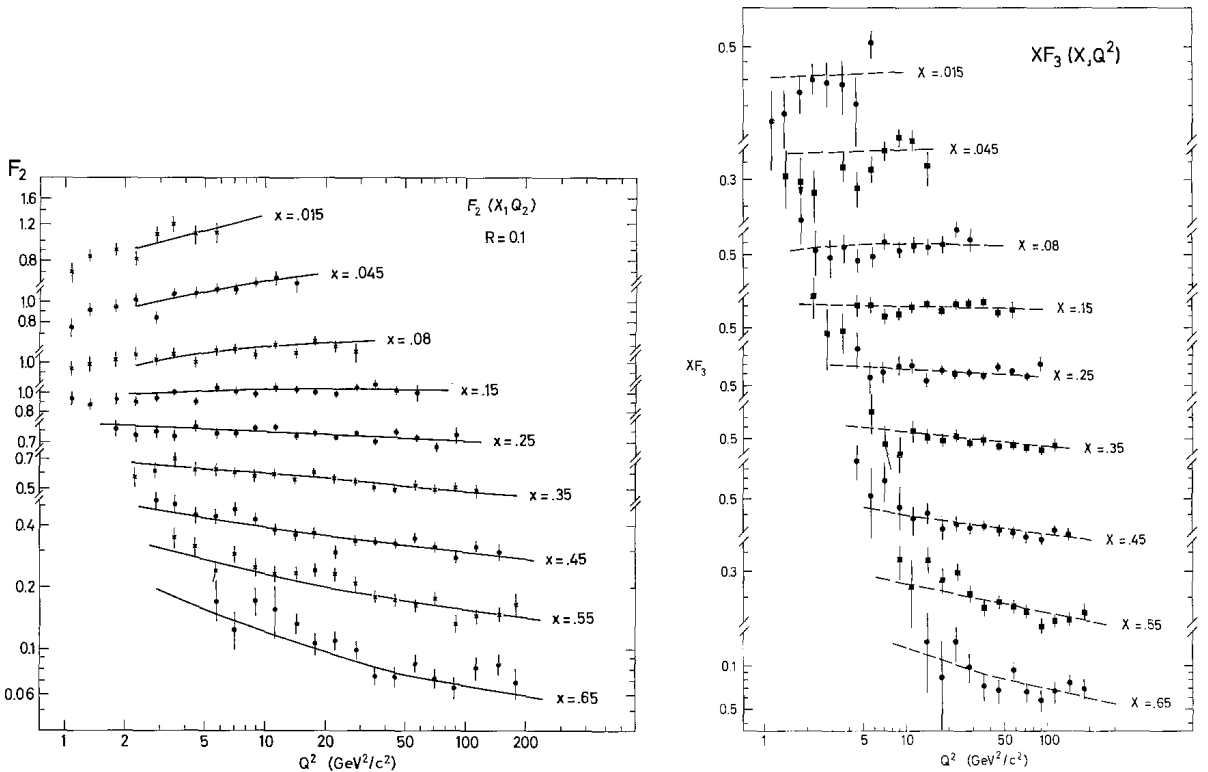

Fig. 22. CDHS results on the structure functions $F_{2}\left(x, Q^{2}\right)$ and $x F_{3}\left(x, Q^{2}\right){ }^{32}$ Lines are fits of the DGLAP evolution equations for $\lambda_{\mathrm{QCD}}=250 \mathrm{MeV}$.

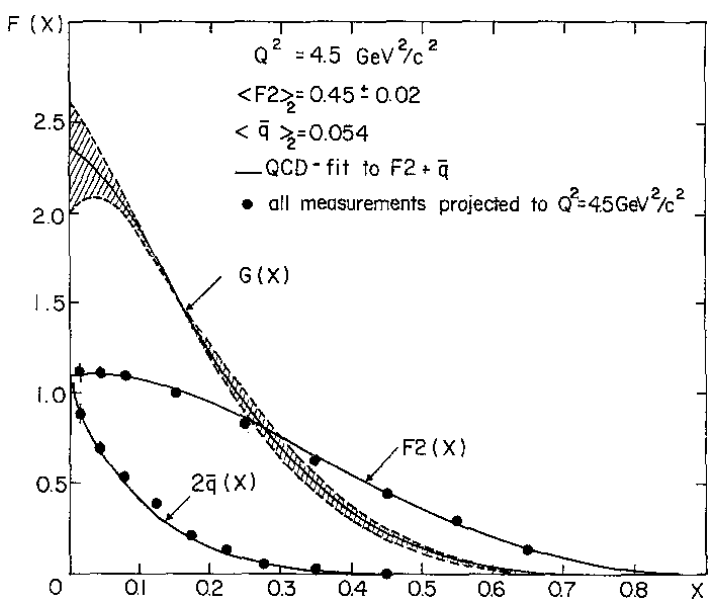

Fig. 23. CDHS results on the gluon function, $g(x),{ }^{34}$ extracted from QCD fits to $F_{2}$ and $\bar{q}$.

to scaling violations of the structure functions. A combined analysis of $F_{2}(x)$ and the antiquark distribution $\bar{q}(x)$ extracted from antineutrino data at large $y$ did allow a simultaneous extraction of the $x$-distribution of the gluon function and $\lambda_{\mathrm{QCD}}$ by fits of the QCD evolution equations. The results are shown in Fig. 23 for the CDHS 

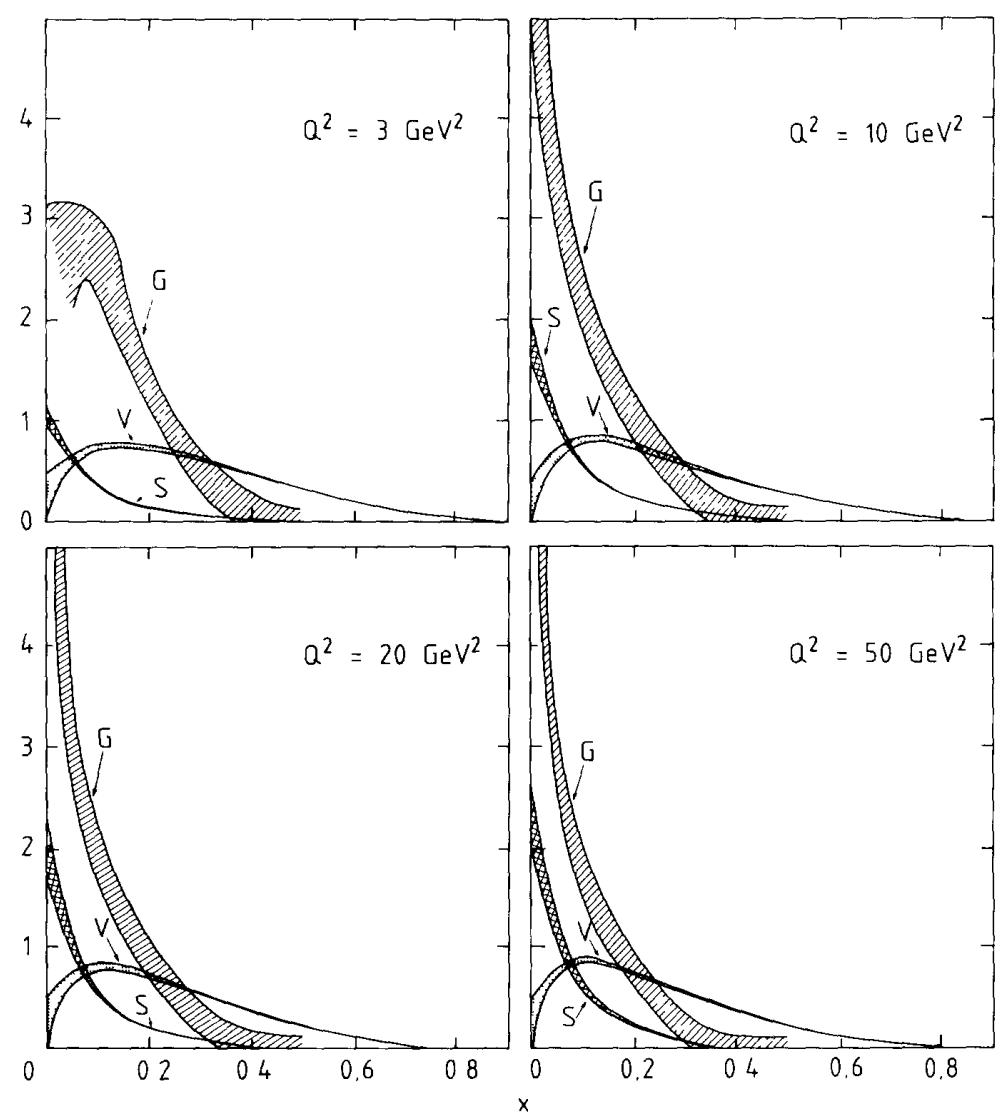

Fig. 24. CHARM results on $g(x),{ }^{35}$ by fitting $F_{2}, x F_{3}$ and $\bar{q}$ at $\operatorname{different} Q^{2}$ values.

analysis at $Q^{2}=4.5 \mathrm{GeV}^{2} / c^{2}, 34$ and in Fig. 24 for the CHARM analysis at several $Q^{2}$ values. ${ }^{35}$ These were the first determinations of the $x$ dependence of the gluon function.

\section{Epilogue}

The first phase of neutrino scattering experiments at the SPS lasted about a decade, culminating in quantitative tests of QCD by means of precise measurements the nucleon structure functions. The CCFR neutrino experiment at Fermilab ${ }^{36}$ with a neutrino beam of $600 \mathrm{GeV}$ energy continued these measurements. The detector used the wide band mixed $\nu_{\mu}$ and $\bar{\nu}_{\mu}$ beam. Due to the rising cross-section the measurements of the structure functions $F_{2}$ and $x F_{3}$ were statistically much more powerful. Similarly, the muon nucleon scattering experiment at the SPS, BCDMS, became leading in the $F_{2}$ measurements. 
After these successful tests of the Standard Model, the interest in neutrino physics moved to the search for neutrino oscillations. Two new experiments were built at CERN, the CERN Hybrid Oscillation Research apparatUS, CHORUS (1993/97) and the Neutrino Oscillation MAgnetic Detector, NOMAD (1995/98). Unfortunately, their sensitivity was not high enough to observe neutrino oscillations. Finally, the first strong experimental evidence for atmospheric neutrino oscillations was announced in 1998 by the Super-Kamiokande experiment in Japan. ${ }^{37}$ This was the first experimental observation demonstrating that the neutrino has non-zero mass. Now, at the forefront of neutrino oscillation research is the Daya Bay Nuclear Reactor experiment in China with their latest $5 \sigma$ measurement of the mixing angle between the first and third generation of neutrinos, $\sin ^{2}\left(2 \theta_{13}\right) \cdot{ }^{38}$

The legacy of the high energy neutrino experiments at CERN remains the precise confirmation of the Quark Parton Model, the precise measurement of the Weinberg angle and the first quantitative tests of QCD with the observation of "scaling violations" in the $Q^{2}$ evolution of the nucleon structure functions and the determination of the QCD interaction strength.

Since then, the ultimate tests of the Standard Model were performed at the $e^{+} e^{-}$collider LEP at CERN and the ep collider HERA at DESY and more recently culminating in the discovery of the Higgs boson at the LHC in 2012.

\section{References}

1. F. J. Hasert et al. (Gargamelle), Phys. Lett. B 46, 121 (1973); 46, 138 (1973).

2. D. Haidt, The Discovery of Weak Neutral Currents, in this book, pp. 165-183.

3. D. H. Perkins, PoS HEP2001, 305 (2001).

4. J. Steinberger, Annals of Physics 327, 3182 (2012).

5. J. D. Bjorken and E. A. Paschos, Phys. Rev. 185, 1975 (1969); R. P. Feynman, Photon Hadron Interactions (Benjamin, NewYork, 1972).

6. J. I. Friedman, Eur. Phys. J. H 36, 469 (2011).

7. T. Eichten et al. (Gargamelle), Phys. Lett. B 46, 274 (1973).

8. H. Deden et al. (Gargamelle), Nucl. Phys. B 85, 269 (1975).

9. G. Miller et al. (SLAC-MIT), Phys. Rev. D 5, 528 (1972).

10. I. Budagov et al. Phys. Lett. B 30, 364 (1969).

11. J. H. Mulvey, Nucl. Phys. B (Proc. Suppl). 36, 427 (1994).

12. D. Haidt, and H. Pietschmann, Electroweak Interactions. Experimental Facts and Theoretical Foundation, Landolt-Börnstein Elementary Particles, Nuclei and Atoms, Vol. 10, 146 (Springer, Berlin, 1988).

13. M. Holder et al. (CDHS), Nucl. Instrum. Meth. 148, 235 (1978).

14. A. N. Diddens et al. (CHARM), Nucl. Instrum. Meth. 178, 27 (1980); C. Bosio et al., Nucl. Instrum. Meth. 157, 35 (1978).

15. T. Eichten et al. Phys. Lett. B 46, 281 (1973).

16. P. C. Bosetti et al. Phys. Lett. B 70, 273 (1977).

17. P. C. Bosetti et al. (BEBC), Nucl. Phys. B 142, 1 (1978). 
18. H. Abramowicz et al. Z. Phys. C 17, 283 (1983).

19. J. J. Aubert et al. (EMC), Phys. Lett. B 105, 322 (1981).

20. A. Bodek et al. Phys. Rev. D 20, 1471 (1979).

21. J. G. H. de Groot et al. (CDHS), Z. Phys. C 1, 143 (1979).

22. M. Holder et al. (CDHS), Phys. Lett. B 71, 222 (1977).

23. M. Jonker et al. (CHARM), Phys. Lett. B 99, 265 (1981).

24. H. Abramowicz et al. (CDHS), Phys. Rev. Lett. 57, 298 (1986).

25. J. V. Allaby (CHARM), Phys. Lett. B 177, 446 (1986).

26. F. Eisele, Rep. Prog. Phys. 49, 233 (1986).

27. J. F. Wheater and C. H. Llewellyn Smith, Nucl. Phys. B 208, 27 (1982); D. Yu. Bardin and O. M. Fedorenko, Sov. J. Nucl. Phys. 30, 418 (1979); D. Yu. Bardin, P. Ch. Christova and O. M. Fedorenko, Nucl. Phys. B 197, 1 (1982); A. Sirlin and W. J. Marchiano, Nucl. Phys. B 189, 442 (1981).

28. S. L. Glashow, J. Iliopoulos, L. Maiani, Phys. Rev. D 2, 1285 (1970).

29. H. Abramowicz et al. (CDHS), Z. Phys. C 15, 19 (1982).

30. J. D. Bjorken, Phys. Rev. D 17, 171 (1978); M. Suzuki, Phys. Lett. B 71, 189 (1977).

31. J. G. H. de Groot et al. (CDHS), Z. Phys. C 1, 143 (1979).

32. H. Abramowicz et al. (CDHS), Z. Phys. C 17, 283 (1983).

33. Y. L. Dokshitzer, Sov. Phys. JETP 46, 641 (1977); V. N. Gribov, L. N. Lipatov, Sov. J. Nucl. Phys. 15, 675 (1972); V. N. Gribov, L .N. Lipatov, Sov. J. Nucl. Phys. 15, 438 (1972); G. Altarelli, G. Parisi, Nucl. Phys. B 126, 298 (1979).

34. H. Abramowicz et al. (CDHS), Z. Phys. C 12, 289 (1982).

35. F. Bergsma et al. (CHARM), Phys. Lett. B 123, 269 (1983).

36. E. Oltman et al. (CCFR), Z. Phys. C 53, 51 (1992).

37. Y. Fukuda et al. (Super-Kamiokande), Phys. Rev. Lett. 81, 1562 (1998).

38. F. P. An et al. (Daya Bay), Phys. Rev. Lett. 112, 061801 (2014). 